\title{
Low-noise polymeric nanomechanical biosensors
}

\author{
Calleja, M.; Tamayo, J.; Nordström, Maria; Boisen, Anja
}

Published in:

Applied Physics Letters

Link to article, DOI:

$10.1063 / 1.2187437$

Publication date:

2006

Document Version

Publisher's PDF, also known as Version of record

Link back to DTU Orbit

Citation (APA):

Calleja, M., Tamayo, J., Nordström, M., \& Boisen, A. (2006). Low-noise polymeric nanomechanical biosensors. Applied Physics Letters, 88(11), 113901. https://doi.org/10.1063/1.2187437

\section{General rights}

Copyright and moral rights for the publications made accessible in the public portal are retained by the authors and/or other copyright owners and it is a condition of accessing publications that users recognise and abide by the legal requirements associated with these rights.

- Users may download and print one copy of any publication from the public portal for the purpose of private study or research.

- You may not further distribute the material or use it for any profit-making activity or commercial gain

- You may freely distribute the URL identifying the publication in the public portal

If you believe that this document breaches copyright please contact us providing details, and we will remove access to the work immediately and investigate your claim 


\title{
Low-noise polymeric nanomechanical biosensors
}

\author{
Montserrat Calleja ${ }^{\text {a) }}$ and Javier Tamayo \\ Bionanomechanics Lab, IMM-Centro Nacional de Microelectronica, Consejo Superior de Investigaciones \\ Cientificas, Isaac Newton 8, 28760 Tres Cantos, Spain \\ Maria Nordström and Anja Boisen \\ Department of Microtechnology and Nanotechnology, DTU, Bldg. 345, DK-2800, Lyngby, Denmark
}

(Received 26 July 2005; accepted 17 February 2006; published online 14 March 2006)

\begin{abstract}
A sensor device based on a single polymer cantilever and optical readout has been developed for detection of molecular recognition reactions without the need of a reference cantilever for subtraction of unspecific signals. Microcantilevers have been fabricated in the photoresist SU-8 with one surface passivated with a thin fluorocarbon layer. The SU-8 surface is sensitized with biological receptors by applying silanization methods, whereas the fluorocarbon surface remains inert to these processes. The thermal and mechanical properties of the chosen materials allow overcoming the main limitations of gold-coated silicon cantilevers: the temperature, $p \mathrm{H}$, and ionic strength cross sensitivities. This is demonstrated by comparing the response of SU-8 cantilevers and that of gold-coated silicon nitride cantilevers to variations in temperature and $p \mathrm{H}$. The sensitivity of the developed polymeric nanomechanical sensor is demonstrated by real-time detection of the human growth hormone with sensitivity in differential surface stress of about $1 \mathrm{mN} / \mathrm{m}$. (C) 2006 American Institute of Physics. [DOI: 10.1063/1.2187437]
\end{abstract}

Nanomechanical biosensors translate molecular recognition events into a nanomechanical response by the use of microcantilever structures. ${ }^{1}$ The underlying physical principle is the buildup of differential surface stress between opposite cantilever sides induced by the attachment of the targeted molecules to one of the cantilever sides, previously sensitized with the corresponding bioreceptor. The advantages of nanomechanical biosensors include real-time labelfree detection, high sensitivity, small sensor area, and capability for simultaneous detection of different targets by use of cantilever arrays. This recent kind of biosensor has demonstrated a high capability for detection of proteins and nucleic acids. $^{2-4}$

Usually, silicon or silicon nitride cantilevers are used, which are coated with a thin gold layer on one side, for selective sensitization of the gold surface with the bioreceptors by well-known self-assembly chemistry. The main limitation of these cantilevers is the signal cross talk between the molecular recognition signal and the environmental changes due to the fluctuations in temperature and ion concentration in aqueous solutions. The difference in the thermal expansion coefficients between gold and silicon (or silicon nitride) produces a deflection of about $20-100 \mathrm{~nm} / \mathrm{K}$ for standard cantilever dimensions. ${ }^{5}$ On the other hand, silicon surfaces are very reactive to ions present in aqueous solutions. ${ }^{6}$ Thus, small fluctuations or drift in the environment properties often obscure the surface stress signals that arise from the molecular recognition events, because these can be as small as $1-10 \mathrm{mN} / \mathrm{m}$, equivalent to a deflection of $1-10 \mathrm{~nm}$ in standard microcantilevers. ${ }^{2,7,8}$ Cross sensitivities can be reduced or eliminated by using cantilever arrays for measuring the differential cantilever bending with respect to a reference cantilever, whose surface must be passive to the target molecules. 2,9 However, this technique is limited by (i) the difficulties for passivation of cantilevers close to the active

${ }^{a)}$ Electronic mail: mcalleja@imm.cnm.csic.es cantilevers in a miniaturized array and (ii) deviations in mechanical properties between reference and measurement cantilevers. ${ }^{9}$

We have fabricated, characterized, and applied for biological detection microcantilevers fabricated in the polymer SU-8 and passivated with a thin fluorocarbon film on one side at the fabrication stage. Both materials have similar thermal and mechanical properties and are relatively inert to the ions present in aqueous solutions. In addition, polymeric cantilevers have the potential for higher sensitivity than silicon and silicon nitride cantilevers due to the 50 times lower Young's modulus. ${ }^{10}$ These characteristics allow for specific biological detection with a single microcantilever.

The fabrication process of the cantilever sensors is based on spin coating of the photosensitive polymer SU-8 (Microchem Corp., Newton, MA) and near-ultraviolet exposure. ${ }^{10} \mathrm{~A}$ silicon wafer is used during the process as support for the multiple SU-8 layers. First, a fluorocarbon film is deposited onto this wafer by induced polymerization in a $\mathrm{C}_{4} \mathrm{~F}_{8}$ plasma. ${ }^{11}$ The purpose of this process step is twofold. On one hand, the thin film serves to easily release the devices from the support silicon wafer. On the other hand, the fluorocarbon film acts as the passive layer due to its chemical inertness. A scanning electron microscopy image of the finished device is shown in Fig. 1. Cantilever deflection measurements were carried out by using the optical beam deflection method. ${ }^{12}$ Reflectivity of uncoated polymer cantilevers is sufficient to perform sensitive deflection measurements with the technology commonly used for gold-coated cantilevers.

In Fig. 2, the nanomechanical response of a flurocarboncoated SU-8 polymer cantilever and that of a gold-coated silicon nitride cantilever (Olympus Ltd., Japan) to temperature variations are compared. For adequate comparison, cantilever dimensions are such that both cantilevers experience similar bending with respect to changes in surface stress. In Table I, $Z_{\sigma}$ accounts for the calculated deflection for a differential surface stress of $1 \mathrm{mN} / \mathrm{m}$ according to Stoney's 


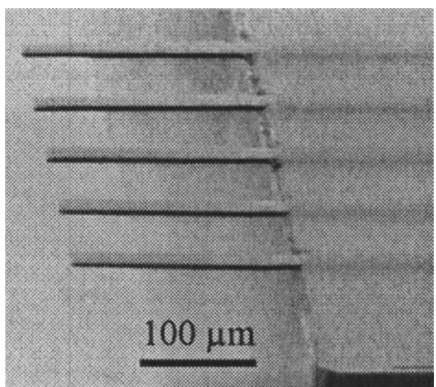

FIG. 1. Scanning electron microscopy image of an array of $200 \mu \mathrm{m}$ long cantilevers fabricated in the polymer SU-8. Cantilever width and thickness are 20 and $4.5 \mu \mathrm{m}$, respectively.

equation, ${ }^{1} Z_{\sigma}=3\left[\sigma(1-v) L^{2}\right] / E t^{2}$, where $E$ is the Young's modulus, $v$ is the Poisson ratio, $t$ and $L$ are thickness and length of the beam, and $\sigma$ accounts for the differential surface stress. The experiment shows that the temperature sensitivity of the polymeric cantilevers is 14 times smaller than that of gold-coated silicon nitride cantilevers. The measured bending compares well to the expected values according to the Timoshenko formulas ${ }^{13}$ described in Eq. (1), considering the dimensions and material properties ${ }^{14,15}$ of the cantilevers, summarized in Table I,

$$
Z=\frac{\frac{3 L^{2}\left(\alpha_{1}-\alpha_{2}\right) \Delta T}{\left(t_{1}+t_{2}\right)}\left(1+\frac{t_{1}}{t_{2}}\right)^{2}}{3\left(1+\frac{t_{1}}{t_{2}}\right)^{2}+\left(1+\frac{t_{1} E_{1}}{t_{2} E_{2}}\right)\left(\frac{t_{1}^{2}}{t_{2}^{2}}+\frac{t_{2} E_{2}}{t_{1} E_{1}}\right)} .
$$

In Eq. (1), $\alpha_{1}$ and $\alpha_{2}$ describe the thermal expansion coefficients of the materials and $\Delta T$ describes the temperature variation. The thermal stability gained by the use of polymer materials is crucial to decrease the noise during bioassays, while keeping a high sensitivity to molecule adsorption. In addition, it allows the application of cantilever sensors for temperature-controlled bioassays. ${ }^{9}$

In order to characterize the cantilever response to variations in the ion concentration in buffer solutions, we have characterized the response of the polymeric cantilevers to $p \mathrm{H}$ variations and compared it to the response of standard goldcoated silicon nitride cantilevers (Fig. 3). Here, a polymeric cantilever activated on one side with a silane layer was used.

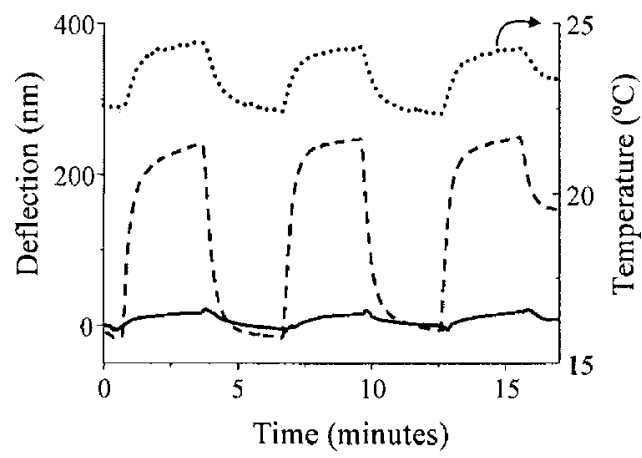

FIG. 2. Experimental curves of the cantilever deflection (left axis) as a function of time when small temperature variations (dotted line, right axis) are induced by means of a Peltier cell placed near the cantilever. The dashed line represents the deflection response of a $200 \mu \mathrm{m}$ long, $40 \mu \mathrm{m}$ wide, and $0.8 \mu \mathrm{m}$ thick silicon nitride cantilever coated with a $20 \mathrm{~nm}$ thick gold layer. The full line represents the deflection response of a fluorocarbon passivated polymeric cantilever, as those shown in Fig. 1. polymeric cantilever, as those shown in Fig. 1.
Downloaded 17 Jun 2010 to 192.38 .67 .112 . Redistribution subject to AlP license or coprs.
TABLE I. Dimensions and material properties of the cantilevers together with the corresponding measured $\left(Z_{m}\right)$ and calculated $\left(Z_{c}\right)$ deflections for a temperature change of $2{ }^{\circ} \mathrm{C} . Z_{\sigma}$ is the calculated deflection for the a differential surface stress of $1 \mathrm{mN} / \mathrm{m}$. The cantilever length is $200 \mu \mathrm{m}$.

\begin{tabular}{lcccccc}
\hline \hline Mateirals & $\begin{array}{c}\text { Thickness } \\
(\mu \mathrm{m})\end{array}$ & $\begin{array}{c}E \\
(\mathrm{GPa})\end{array}$ & $\begin{array}{c}\alpha \\
\left(10^{-6} / \mathrm{K}\right)\end{array}$ & $\begin{array}{c}Z_{c} \\
(\mathrm{~nm})\end{array}$ & $\begin{array}{c}Z_{m} \\
(\mathrm{~nm})\end{array}$ & $\begin{array}{c}Z_{\sigma} \\
(\mathrm{nm})\end{array}$ \\
\hline $\mathrm{Si}_{3} \mathrm{~N}_{4}$ & 0.8 & 280 & 2.6 & & & \\
$\mathrm{Au}$ & 0.02 & 7.5 & 14.2 & 240 & 255 & 0.5 \\
& & & & & & \\
$\mathrm{FC}^{\mathrm{a}}$ & 0.02 & 8 & $34-102$ & & & \\
$\mathrm{SU}-8$ & 4.5 & 5.4 & 52 & $6-16$ & 18 & 0.9 \\
\hline \hline
\end{tabular}

${ }^{\mathrm{a}}$ Flouorocarbon film.

For a $p \mathrm{H}$ change from 7 to 11 , the silicon nitride cantilever response was six times larger than that for the SU-8 cantilever. When the $p \mathrm{H}$ was decreased from $p \mathrm{H} 7$ to $p \mathrm{H} \mathrm{2,} \mathrm{the}$ silicon nitride cantilever undergoes a bending 50 times larger than that of the polymeric cantilever. The polymeric materials provide enhanced stability to variations in the ion concentration that could screen the molecular recognition signal.

The polymeric sensors were applied for measuring the immunoreaction between the human growth hormone (hGH) and its antibody ( $\alpha$-hGH). Previously, the gold-coated silicon nitride cantilevers were applied and the immunospecific binding of $\alpha$-hGH could not be detected. A protocol specially developed for SU-8 was applied for immobilization of the human growth hormone antigen. Briefly, SU-8 cantilevers were immersed into $1: 3, \mathrm{H}_{2} \mathrm{SO}_{4}: \mathrm{H}_{2} \mathrm{O}_{2}$ for $10 \mathrm{~s}$ and washed in MilliQ water afterwards. Under this treatment, epoxy groups on the SU-8 surface open up due to the highly acidic environment and react with oxygen leaving hydroxyl groups on the surface. The hydroxyl groups serve as binding sites for the formation of self-assembled silane monolayers when the cantilevers are incubated in mercaptosilane solution for $1 \mathrm{~h}$. The thiol-ended silane monolayer is then activated by the heterobifunctional crosslinkers $N$-hydroxysuccinimide and $N$-(3-dimethylaminopropyl)- $N^{\prime}$-ethylcarbodiimide hydrochloride (from Sigma-Aldrich Co.). The immobilization of the hGH antigen at a concentration of $5 \mu \mathrm{g} / \mathrm{ml}$ was performed under flow conditions in sodium phosphate buffer $(p \mathrm{H}=7.3)$ and monitored in real-time [marked as (1) in Fig. 4]. The hGH adsorption produced a cantilever deflection of about $14 \mathrm{~nm}$ toward the fluorocarbon film, which corre-

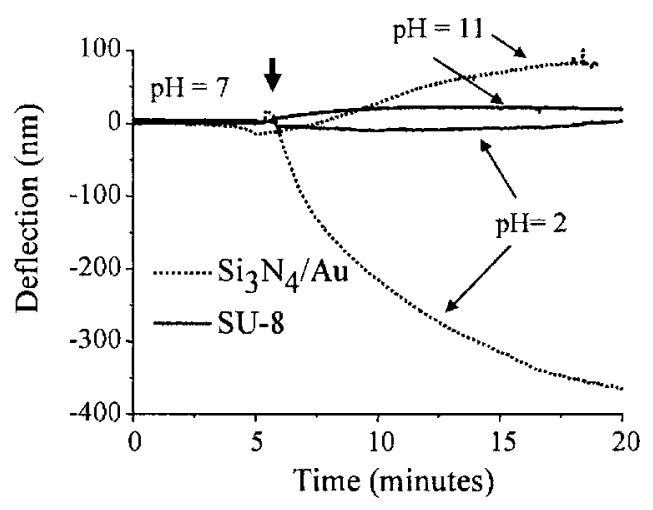

FIG. 3. Nanomechanical response to $p \mathrm{H}$ variations in solution. The dotted lines correspond to the response of a commercial gold-coated silicon nitride cantilever, while the full lines correspond to the bending of a polymeric cantilever for the same $p \mathrm{H}$ variations. The arrow indicates the moment when 


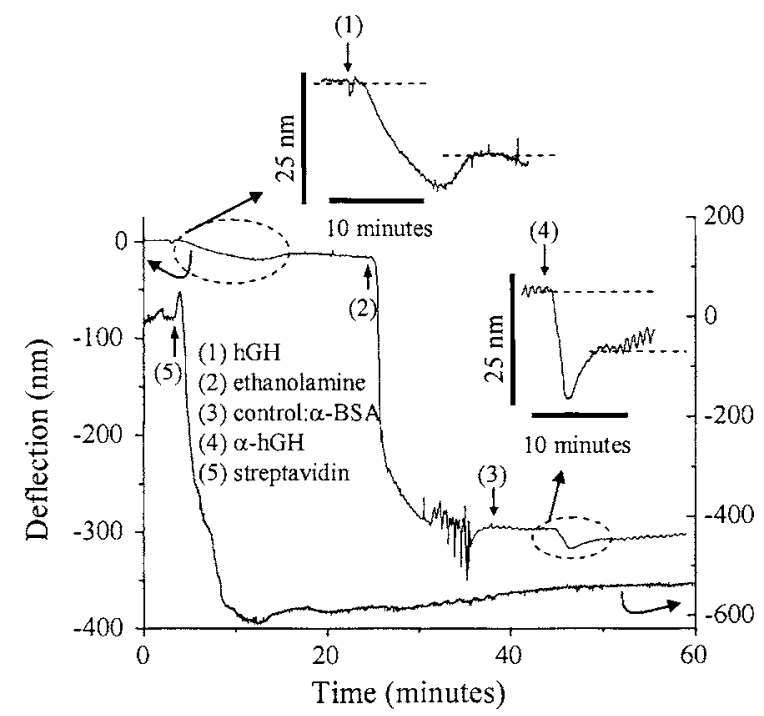

FIG. 4. The graph shows two measurements of molecular recognition events. The upper curve corresponds to a full experiment for real-time detection of human growth hormone with its specific antibody. The deflection signals corresponding to the immobilization of the antigen hGH (1) and the reaction with the monoclonal antibody $\alpha$-bGH (4) have been enlarged for major clarity. Ethanolamine immobilization (2) was performed after antigen immobilization to block the remaining binding sites that could give unspecific adsorption signals. The control experiment by exposure of the cantilever to $\alpha$-BSA is labeled as (3). The lower curve corresponds to the molecular recognition reaction between streptavidin (5) and biotinilated BSA, which is covalently anchored to a polymeric cantilever.

sponds to a differential surface stress of $16 \mathrm{mN} / \mathrm{m}$. A small decrease in the deflection signal is observed during the buffer rinsing due to the detachment of the loosely bound molecules. The observed permanent bending accounts for the differential surface stress due to the covalent immobilization of hGH on the SU-8 surface. Afterwards, $1 \mathrm{mM}$ ethanolamine was injected into the flow cell [marked as (2) in Fig. 4]. Due to its smaller size and high reactivity, this molecule blocks all the possible remaining binding sites on the silane layer to avoid unspecific adsorption during injection of the monoclonal antibody $(\alpha-\mathrm{hGH})$. The binding of ethanolamine produces a large deflection of the cantilever, corresponding to a differential surface stress of $329 \mathrm{mN} / \mathrm{m}$. As a control experiment, the cantilever was first exposed to an unspecific antibody ( $\alpha$-BSA, antibody to bovine serum albumin) at a concentration of $5 \mu \mathrm{g} / \mathrm{ml}$ [marked as (3) in Fig. 4]. We do not observe a cantilever response, indicating that no attachment of $\alpha$-BSA occurred. After the positive control, the specific antibody to $\mathrm{hGH}$ at a concentration of $5 \mu \mathrm{g} / \mathrm{mL}$ was flowed over the cantilever [marked as (4) in Fig. 4]. The molecular recognition between the $\alpha$-hGH and its antigen gave rise to a permanent bending of $11 \mathrm{~nm}$, corresponding to a differential surface stress of $13 \mathrm{mN} / \mathrm{m}$.

The reliability of the polymeric sensor was also validated by detecting the molecular recognition between biotin and streptavidin. Biotinilated BSA was covalently attached on the SU-8 side of the cantilever by using the same chem- istry as for hGH immobilization. BSA acts as a vertical spacer, making biotin binding sites easily accessible to streptavidin. Exposure of the cantilever to streptavidin at a concentration of $5 \mu \mathrm{g} / \mathrm{ml}$ gave rise a differential surface stress of about $680 \mathrm{mN} / \mathrm{m}$ [marked as (5) in Fig. 4].

In conclusion, the feasibility of a biosensor device based on optical readout of a single cantilever sensor fully fabricated in polymeric materials has been demonstrated. The mechanical properties of the polymer material allow for high sensitivity. In addition, the passivation of one side of the cantilever with a fluorocarbon film, together with the immobilization protocol developed, has demonstrated good specificity to the reactions of interest in several different measurements. Furthermore, the materials chosen for the cantilever preserve the stability of the measurements with respect to thermal changes or electrolyte fluctuations in solution. This allows for detection of differential surface stresses as small as $1 \mathrm{mN} / \mathrm{m}$ without the need for a reference cantilever. Further improvements of the fabrication process should allow reducing the cantilever thickness by at least a factor of 5 , leading to sensitivities of about $50 \mu \mathrm{N} / \mathrm{m}$. This development also has great potential for application in temperaturecontrolled studies of biomolecules or polymerase chain reaction thermal cycling experiments.

The authors acknowledge J. M. Rodríguez-Prado and M. Mellado for providing reagents for the molecular recognition experiments and J. Treviño, E. Mauriz, and D. Haefliger for fruitful discussion. J.T. and M.C. acknowledge financial support from the Spanish Ministry of Science (MERG-CT-2004510638) and the European Union (GEN2001-4856-C13-11).

${ }^{1}$ R. Raiteri, M. Grattarola, H.-J. Butt, and P. Skládal, Sens. Actuators B 79, 115 (2001).

${ }^{2}$ R. McKendry, J. Y. Zhang, Y. Arntz, T. Strunz, M. Hegner, H. P. Lang, M. K. Baller, U. Certa, E. Meyer, H. J. Guntherodt, and C. Gerber, Proc. Natl. Acad. Sci. U.S.A. 99, 9783 (2002).

${ }^{3}$ G. Wu, R. H. Datar, K. M. Hansen, T. Thundat, R. J. Cote, and A. Majumdar, Nat. Biotechnol. 19, 856 (2001).

${ }^{4}$ M. Álvarez, A. Calle, J. Tamayo, L. M. Lechuga, A. Abad, and A. Montoya, Biosens. Bioelectron. 18, 649 (2003).

${ }^{5}$ J. R. Barnes, R. J. Stephenson, C. N. Woodburn, S. J. O'Shea, M. E. Welland, T. Rayment, J. K. Gimzewski, and Ch. Gerber, Rev. Sci. Instrum. 65, 3793 (1994).

${ }^{6}$ H.-F. Ji, K. M. Hansen, Z. Hu, and T. Thundat, Sens. Actuators B 72, 233 (2001).

${ }^{7}$ M. Álvarez, L. G. Carrascosa, M. Moreno, A. Calle, A. Zaballos, L. M. Lechuga, C. Martínez-A, and J. Tamayo, Langmuir 20, 9663 (2004).

${ }^{8}$ H. J. Butt, J. Colloid Interface Sci. 180, 251 (1996).

${ }^{9}$ R. Marie, J. Thaysen, C. B. V. Christensen, and A. Boisen, Microelectron. Eng. 67-68, 893 (2003).

${ }^{10}$ M. Calleja, J. Tamayo, A. Johansson, P. Rasmussen, L. M. Lechuga, and A. Boisen, Sens. Lett. 1, 20 (2003).

${ }^{11}$ D. Haefliger, M. Nordström, P. A. Rasmussen, and A. Boisen, Microelectron. Eng. 78-79, 88 (2005).

${ }^{12}$ M. Calleja, M. Nordstrom, M. Alvarez, J. Tamayo, L. M. Lechuga, and A. Boisen, Ultramicroscopy 105, 215 (2005).

${ }^{13}$ N. V. Lavrik, M. J. Sepaniak, and P. G. Datskos, Rev. Sci. Instrum. 75, 2229 (2004).

${ }^{14}$ M. A. Butler, R. J. Buss, and A. Galuska, J. Appl. Phys. 70, 2326 (1991).

${ }^{15}$ G. Tang, X. Ma, M. Sun, and X. Li, Carbon 43, 345 (2005). 